\title{
Applications of an elementary resolution of singularities algorithm to exponential sums and congruences modulo $p^{n}$
}

\author{
Michael Greenblatt
}

December 9, 2014

\section{Introduction and statement of results}

In this paper, we use the resolution of singularities algorithm of [G4] to generalize to arbitrary local fields of characteristic zero the theorems of [G3] on $\mathbf{R}^{n}$ sublevel set volumes and oscillatory integrals with real phase function. The proofs of these generalizations use various aspects of the resolution of singularities algorithms of [G4] (but for the most part not the actual resolution of singularities theorems themselves.) The $p$-adic cases of our results provide new estimates for exponential sums as well as new bounds on how often a function $f(x)$ such as a polynomial with integer coefficients is divisible by various powers of a prime $p$ when $x$ is an integer. Thus we use classical analysis resolution of singularities methods on a class of problems traditionally approached using toric resolution of singularities techniques.

The estimates of this paper for the sublevel set measures and oscillatory integral decay rates will as in [G3] be expressed in terms of properties of the Newton polyhedron of the phase function $f(x)$. Such estimates go back to [V] and for $p$-adic fields there is an extensive body of research on such estimates. We mention [D][DHo] $[\mathrm{DLo}][\mathrm{LM}][\mathrm{Ve}][\mathrm{Wr}][\mathrm{Zu} 1][\mathrm{Zu} 2]$ for a sampling. Unlike in many such papers, especially in higher dimensions, we will not require a nondegeneracy condition such as that of [V]. Instead, as in [G3] our theorems will be stated in terms of the orders of the zeroes of certain polynomials $f_{F}(x)$ associated to $N(f)$, given in Definition 1.5 below, and will go beyond such a nondegeneracy condition. We will have results for any Newton distance (see Definiton 1.6), and the Newton distance will determine the conditions required on the maximal order of the zeroes of the above polynomials. Generalizations when the Newton distance is less than or equal to 1 will be discussed after the statement of Theorem 1.2.

In this paper, a real oscillatory integral denotes an expression

$$
I(\lambda)=\int_{\mathbf{R}^{n}} e^{i \lambda f(x)} \phi(x) d x
$$

Here $\phi(x)$ is a cutoff function defined on an appropriately small bounded open set con1001070

This research was supported in part by NSF grants DMS-0919713 and DMS- 
taining the origin. Using resolution of singularities it can be shown that if $\nabla f(0)=0$ (the nontrivial case), then $I(\lambda)$ has an asymptotic expression as $\lambda \rightarrow \infty$ of the form

$$
I(\lambda)=c_{\phi} e^{i \lambda f(0)}(\ln (\lambda))^{m} \lambda^{-\delta}+o\left((\ln (\lambda))^{m} \lambda^{-\delta}\right)
$$

Here $m$ is a nonnegative integer and $\delta>0$, both independent of $\phi$, and given any sufficiently small neighborhood $U$ of the origin $c_{\phi}$ is nonzero for at least one $\phi$ supported in $U$.

To understand what the complex analogue of (1.1) might be, note that a key characteristic of oscillatory integrals (1.1) is that for any $\lambda$ the function $e^{i \lambda t}$ is a (continuous) additive character on $\mathbf{R}$. Furthermore, all continuous additive characters of $\mathbf{R}$ are of this form. Thus with the view of finding analogues to (1.1) we are interested in what the continuous additive characters of $\mathbf{C}$ are. Suppose $\chi(z)$ is one such character. Then $\chi(z)=\chi(\operatorname{Re}(z), 0) \chi(0, \operatorname{Im}(z))=e^{i \lambda_{1} \operatorname{Re}(z)} e^{i \lambda_{2} \operatorname{Im}(z)}$ for some $\lambda_{1}$ and $\lambda_{2}$. There necessarily exists some complex number $w$ such that for any $z, \operatorname{Re}(w z)=\lambda_{1} \operatorname{Re}(z)+\lambda_{2} \operatorname{Im}(z)$. Thus the continuous additive characters of $\mathbf{C}$ are the functions of the form $e^{i \operatorname{Re}(w z)}$ for some $w \in \mathbf{C}$. Hence for our purposes natural analogues of (1.1) are the integrals

$$
I(w)=\int_{\mathbf{C}^{n}} e^{i \operatorname{Re}(w f(z))} \phi(z) d z
$$

Here again $\phi(z)$ is a cutoff function, and the goal now will be to find optimal decay estimates $|I(w)| \leq C(\ln |w|)^{m}|w|^{-\delta}$ as $|w| \rightarrow \infty$. As in the real case, if $f(z)$ has some nonvanishing first derivative at the origin then one gets arbitrarily fast decay, so we always assume this is not the case here.

We now direct our attention to characters on $p$-adic fields. Any $p$-adic number $x$ may be written as $x=\sum_{k=k_{0}}^{\infty} b_{k} p^{k}$, where each $b_{k} \in\{0, \ldots, p-1\}, b_{k_{0}} \neq 0$, and $|x|=p^{-k_{0}}$. Addition on the $p$-adics in such a form is done as one adds natural numbers written in base $p$, using carrying. It is well-known (and relatively easy to show) that the continuous additive characters on the $p$-adics are functions of the form $\chi(x)=\xi(y x)$, where $y \in \mathbf{Q}_{p}$ and where $\xi\left(\sum_{k \geq k_{0}} b_{k} p^{k}\right)=e^{2 \pi i\left(\sum_{k=k_{0}}^{-1} b_{k} p^{k}\right)}$ for $k_{0}<0$ and $\xi(x)=1$ for $k_{0} \geq 0$.

One can determine the additive characters on any $p$-adic field (a finite extension field of some $\mathbf{Q}_{p}$ ) from the characters on $\mathbf{Q}_{p}$ analogously to how the additive characters on $\mathbf{C}$ were determined above from those on $\mathbf{R}$. Namely, suppose $K$ is a field extension of $\mathbf{Q}_{p}$ of degree $l$. Then we may write elements $x$ of $K$ in the form $\left(x_{1}, \ldots, x_{l}\right)$ where each $x_{k} \in \mathbf{Q}_{p}$. Thus if $\chi$ is a continuous additive character on $K$, one has

$$
\chi(x)=\chi\left(x_{1}, 0, \ldots, 0\right) \ldots \chi\left(0, \ldots, 0, x_{l}\right)
$$

By the form of the characters on $\mathbf{Q}_{p}$, for some $y_{1}, \ldots, y_{l} \in Q_{p}$ the above can be written as

$$
\chi(x)=\xi\left(y_{1} x_{1}\right) \ldots \xi\left(y_{l} x_{l}\right)=\xi\left(y_{1} x_{1}+\ldots+y_{l} x_{l}\right)
$$


Analogously to the complex case, there is some $z \in K$ such that the first component of $z x$ is $y_{1} x_{1}+\ldots+y_{l} x_{l}$ for all $x \in K$. Denoting this first component by $R(z x)$, for any $x$ one therefore has

$$
\chi(x)=\xi(R(z x))
$$

Thus a natural analogue of the oscillatory integral (1.1) for $p$-adic fields is given by

$$
I(z)=\int_{|x|<\delta} \xi(R(z f(x))) d x
$$

Since all smooth functions on a $p$-adic field are locally constant, instead of having a cutoff function $\phi(x)$ in (1.5) we restrict the domain of integration to $|x|<\delta$ for some $\delta$.

In the real case it is well known (see $[\mathrm{AGV}]$ ) that sharp estimates for oscillatory integrals usually follow from sharp estimates for the measure of sublevel sets; given a sufficiently small open set $U$ containing the origin one may look for the best possible estimate of the form $|\{x \in U:|f(x)|<\epsilon\}|<C|\ln (\epsilon)|^{m} \epsilon^{\delta}$. This $(m, \delta)$ can then be translated into decay estimates for the oscillatory integral (1.1), and this rate of decay is sharp except in certain exceptional situations. This translation is proven using resolution of singularities to show both the sublevel set measures and the oscillatory integral decay have asymptotic expansions, and then using integration by parts in a certain way to go from the sublevel set measures to the oscillatory integral estimates. The analgoue for $p$-adic fields was proved by Igusa [I1]-[I3], and in Theorem 2.2 we will prove the corresponding statement for $K=\mathbf{C}$ using results from [G4].

We now give some relevant definitions.

Definition 1.4. Let $f(x)$ be a function such that $f(x)$ has a convergent power series expansion $\sum_{\alpha} f_{\alpha} x^{\alpha}$ on a neighborhood of the origin in $K^{n}$. For any $\alpha$ for which $f_{\alpha} \neq 0$, let $Q_{\alpha}$ be the octant $\left\{t \in \mathbf{R}^{n}: t_{i} \geq \alpha_{i}\right.$ for all $\left.i\right\}$. Then the Newton polyhedron $N(f)$ of $f(x)$ is defined to be the convex hull of all $Q_{\alpha}$.

A Newton polyhedron can contain faces of various dimensions in various configurations. These faces can be either compact or unbounded. In this paper, as in earlier work such as [G3] and [V], an important role is played by the following functions, defined for compact faces of the Newton polyhedron. A vertex is always considered to be a compact face of dimension zero.

Definition 1.5. Suppose $F$ is a compact face of $N(f)$. Then if $f(x)=\sum_{\alpha} f_{\alpha} x^{\alpha}$ denotes the Taylor expansion of $f$ like above, define $f_{F}(x)=\sum_{\alpha \in F} f_{\alpha} x^{\alpha}$.

The statements of several of our theorems will use the following terminology.

Definition 1.6. Assume $N(f)$ is nonempty. Then the Newton distance $d(f)$ of $f(x)$ is defined to be $\inf \{t:(t, t, \ldots, t, t) \in N(f)\}$. 
Definition 1.7. The central face of $N(f)$ is the face of $N(f)$ of minimal dimension intersecting the line $t_{1}=t_{2}=\ldots=t_{n}$.

In Definition 1.7, the central face of $N(f)$ is well-defined since it is given by the intersection of all faces of $N(f)$ intersecting the line $t_{1}=t_{2}=\ldots=t_{n}$. An equivalent definition that is sometimes used (such as in [AGV]) is that the central face of $N(f)$ is the unique face of $N(f)$ intersecting the line $t_{1}=t_{2}=\ldots=t_{n}$ in its interior.

We now come to our theorems concerning sublevel set measures and oscillatory integrals. They are analogues of corresponding results in [G3]. $f(x)$ will always denote a function with convergent power series on a neighborhood of the origin with $f(0)=0$. The statements of the theorems are slightly different for different fields $K$, in that they will depend on the dimension of $K$ over its base field. Correspondingly, in the following $b_{K}=1$ if $K=\mathbf{R}, b_{K}=2$ if $K=\mathbf{C}$, and for an extension of the $p$-adics $b_{K}$ denotes the degree of $K$ over $\mathbf{Q}_{p}$.

We use the notation $|A|$ to denote the measure of a set $A$. If $K=\mathbf{R}$ or $\mathbf{C}$ this denotes the usual Lebesgue measure. When $K$ is $\mathbf{Q}_{p}$ we use the traditional Haar measure that assigns measure 1 to $\{x:|x| \leq 1\}$, and if $K$ is a finite extension of $\mathbf{Q}_{p}$ we use the product measure induced by that of $\mathbf{Q}_{p}$. As for which valuation we use on $K$, we will use the traditional $|x|_{p}=p^{-v_{p}(x)}$ valuation on $\mathbf{Q} \subset \mathbf{Q}_{p}$ and its natural extension to $K$ if $K$ is a finite extension of $\mathbf{Q}_{p}$. Note that the $b_{K}$ th power of this valuation is used in $[\mathrm{Cl}]$ which accounts for the difference in the exponents in the theorems such as in section 2.

Theorem 1.1. Let $K_{0}$ denote $K-\{0\}$. For a compact face $F$ of $N(f)$, let $o(F)$ denote the maximum order of any zero of $f_{F}(x)$ in $K_{0}^{n}$. Let $h$ denote the dimension of the central face of $N(f)$. For a small open set $U$ containing the origin, let $g(\epsilon)$ denote the measure of $\{x \in U:|f(x)|<\epsilon\}$. Then if $U$ is sufficiently small, there are positive constants $C$ and $C^{\prime}$ depending on $U$ and $f$ such that the following hold for $0<\epsilon<\frac{1}{2}$.

a) If $o(F) \leq d(f)$ for all compact faces $F$ of $N(f)$, with $o(F)<d(f)$ when $F$ is a subset of the central face of $N(f)$, then

$$
C|\ln (\epsilon)|^{n-h-1} \epsilon^{\frac{b_{K}}{d(f)}} \leq g(\epsilon) \leq C^{\prime}|\ln (\epsilon)|^{n-h-1} \epsilon^{\frac{b_{K}}{d(f)}}
$$

b) If $o(F) \leq d(f)$ for all compact faces $F$ of $N(f)$ with $o(F)=d(f)$ for at least one compact $F$ contained in the central face of $N(f)$, then

$$
C|\ln (\epsilon)|^{n-h-1} \epsilon^{\frac{b_{K}}{d(f)}} \leq g(\epsilon) \leq C^{\prime}|\ln (\epsilon)|^{n-h} \epsilon^{\frac{b_{K}}{d(f)}}
$$

c) If $o(F)>d(f)$ for at least one compact face $F$ of $N(f)$, let $s(f)$ denote $\sup _{F} o(F)$. Then we have

$$
C|\ln (\epsilon)|^{n-h-1} \epsilon^{\frac{b_{K}}{d(f)}} \leq g(\epsilon) \leq C^{\prime} \epsilon^{\frac{b_{K}}{s(f)}}
$$


Theorem 1.2. If in Theorem 1.1 one has an upper bound $g(\epsilon) \leq C^{\prime}|\ln (\epsilon)|^{m} \epsilon^{\delta}$, then for sufficiently large $|\lambda|,|w|$, or $|z|$ the oscillatory integral (1.1), (1.3), or (1.5) respectively satisfies the analogous bound $|I(\lambda)| \leq C(\ln |\lambda|)^{m}|\lambda|^{-\delta},|I(w)| \leq C(\ln |w|)^{m}|w|^{-\delta}$, or $|I(z)| \leq C(\ln |z|)^{m}|z|^{-\delta}$.

In the case where $K=\mathbf{R}$, Theorems 1.1 and 1.2 were proven in [G3], where the oscillatory integral upper bounds corresponding to parts a) and b) of Theorem 1.1 were shown typically to be sharp. It is unclear what the sharpness situation is for oscillatory integrals in the $p$-adic case. The upper bounds of part c) of Theorem 1.1 and the corresponding upper bounds of Theorem 1.2 are usually not sharp; to get sharp estimates one normally needs more detailed information about the singularities of $f(x)$ than the Newton polyhedron provides.

It is worth mentioning that there is an additional situation where Theorem 1.2 is known to hold, namely where $d(f) \leq 1$ and $o(F) \leq 1$ for all compact faces $F$ of $N(f)$. This was proved using toric resolution of singularities in [V], and the method contained therein generalizes to the $K$ considered in this paper. Since in this paper Theorem 1.2 is effectively a consequence of Theorem 1.1, where the analogous statement is false, this case is not covered by Theorem 1.2. However, it is possible to prove this case directly using Lemma 3.1 through a direct integration by parts of the exponential since the phase function will have nonvanishing gradient.

Often in papers using the toric methods of [AGV], theorems explicitly make the assumption that

$$
\{x \in U: \nabla f(x)=0\} \subset\{x \in U: f(x)=0\}
$$

One can use resolution of singularities to see that (1.6) always holds in a sufficiently small neighborhood of the origin (when $f(0)=0$ ). Namely, let $\Psi(x)$ be as in Hironaka's theorem such that $f \circ \Psi(x)$ is locally comparable to a monomial; that is, in a local coordinate system $f \circ \Psi(x)$ is of the form $a(x) m(x)$ where $m(x)$ is a monomial and $a(x)$ is nonvanishing. Then by the chain rule, (1.6) holds on a neighborhood of the origin if the analogous statement for $f \circ \Psi$ holds on a neighborhood of $\Psi^{-1}(0)$. The latter statement can be seen to be true by a direct computation in coordinates for which $f \circ \Psi(x)$ is of the this form $a(x) m(x)$.

Thus while we don't explicitly assume (1.6), we do only prove Theorems 1.1 and 1.2 on a neighborhood of the origin that is sufficiently small for our various lemmas to work. Because of the involved nature of the arguments leading to these theorems, it is hard to ascertain if this neighborhood is also sufficiently small that (1.6) necessarily holds throughout.

It is natural to ask to what extent the arguments giving Theorem 1.1 and 1.2 extend to local fields of positive characteristic. The argument in [G4] that proves Lemma 3.5 of that paper (which is the same as Lemma 3.1 is this paper) makes use of the fact that the field has characteristic zero; equation (3.1b) does not immediately hold in the positive characteristic case by the same argument. In addition, we utilize the Weierstrass 
preparation theorem for characteristic zero in the proof of Theorem 2.1, so any extension to the positive characteristic case would require an analogue or a substitute. So any adaptation of our proofs to the positive characteristic case would have to take into account both issues.

\section{Number theoretic consequences.}

The cases $K=\mathbf{Q}_{p}$ of Theorems 1.1 and 1.2 have some number-theoretic consequences. In Theorems 1.3 and $1.4, f(x)$ denotes a power series $\sum f_{\alpha} x^{\alpha}$ with integer coefficients that converges on a neighborhood of the origin when viewed as a power series on $\mathbf{Q}_{p}^{n}$, satisfying $f(0)=0$. The following theorem is a consequence of Theorem 1.1; the condition that each $a_{i}$ is sufficiently large ensures that Theorem 1.1 applies to $f_{a}(x)$ on an entire ball of radius 1 centered at the origin.

Theorem 1.3. For $a=\left(a_{1}, \ldots, a_{n}\right)$, each $a_{i}$ an integer, let $f_{a}\left(x_{1}, \ldots, x_{n}\right)$ denote the function $f\left(p^{a_{1}} x_{1}, \ldots, p^{a_{n}} x_{n}\right)$. There exists a constant $M>0$ depending on $f(x)$ such that if $a_{i}>M$ for all $i$ then there are constants $C, C^{\prime}>0$ depending on $a, f(x)$, and $p$ such that the following hold for every positive integer $l$.

a) If $o(F) \leq d(f)$ for all compact faces $F$ of $N(f)$, with $o(F)<d(f)$ when $F$ is a subset of the central face of $N(f)$, then

$$
C l^{n-h-1} p^{-\frac{l}{d(f)}} \leq \frac{1}{p^{l n}} \#\left\{x \in\left\{0, \ldots, p^{l}-1\right\}^{n}: p^{l} \text { divides } f_{a}(x)\right\} \leq C^{\prime} l^{n-h-1} p^{-\frac{l}{d(f)}}
$$

b) If $o(F) \leq d(f)$ for all compact faces $F$ of $N(f)$ with $o(F)=d(f)$ for at least one compact $F$ contained in the central face of $N(f)$, then

$$
C l^{n-h-1} p^{-\frac{l}{d(f)}} \leq \frac{1}{p^{l n}} \#\left\{x \in\left\{0, \ldots, p^{l}-1\right\}^{n}: p^{l} \text { divides } f_{a}(x)\right\} \leq C^{\prime} l^{n-h} p^{-\frac{l}{d(f)}}
$$

c) If $o(F)>d(f)$ for at least one compact face $F$ of $N(f)$, let $s(f)$ denote $\sup _{F} o(F)$. Then

$$
C l^{n-h-1} p^{-\frac{l}{d(f)}} \leq \frac{1}{p^{l n}} \#\left\{x \in\left\{0, \ldots, p^{l}-1\right\}^{n}: p^{l} \text { divides } f_{a}(x)\right\} \leq C^{\prime} p^{-\frac{l}{s(f)}}
$$

Similarly, the oscillatory integral result Theorem 1.2 implies

Theorem 1.4. Let $f_{a}\left(x_{1}, \ldots, x_{n}\right)$ be as in Theorem 1.3. There exists a constant $M>0$ depending on $f(x)$ such that if $a_{i}>M$ for all $i$ then there is a constant $C>0$ depending on $a, f(x)$, and $p$ such that the following hold for every positive integer $l$.

a) If $o(F) \leq d(f)$ for all compact faces $F$ of $N(f)$, with $o(F)<d(f)$ when $F$ is a subset of the central face of $N(f)$, then

$$
\frac{1}{p^{l n}}\left|\sum_{x \in\left\{0, \ldots, p^{l}-1\right\}^{n}} e^{2 \pi i \frac{f_{a}(x)}{p^{l}}}\right| \leq C l^{n-h-1} p^{-\frac{l}{d(f)}}
$$


b) If $o(F) \leq d(f)$ for all compact faces $F$ of $N(f)$ with $o(F)=d(f)$ for at least one compact $F$ contained in the central face of $N(f)$, then

$$
\frac{1}{p^{l n}}\left|\sum_{x \in\left\{0, \ldots, p^{l}-1\right\}^{n}} e^{2 \pi i \frac{f_{a}(x)}{p^{l}}}\right| \leq C l^{n-h} p^{-\frac{l}{d(f)}}
$$

c) If $o(F)>d(f)$ for at least one compact face $F$ of $N(f)$, let $s(f)$ denote $\sup _{F} o(F)$. Then

$$
\frac{1}{p^{l n}}\left|\sum_{x \in\left\{0, \ldots, p^{l}-1\right\}^{n}} e^{2 \pi i \frac{f_{a}(x)}{p^{l}}}\right| \leq C p^{-\frac{l}{s(f)}}
$$

Since in Theorems 1.3 and 1.4 the constants $C$ and $C^{\prime}$ do depend on $p$, no uniformity in $p$ is proven here such as was conjectured in the homogeneous case by Igusa [I3]. Also note that if $f\left(x^{b_{1}}, \ldots, x^{b_{n}}\right)$ is homogeneous for some positive integers $b_{i}$ then one can automatically replace $f_{a}(x)$ with $f(x)$ in Theorems 1.3 and 1.4 by choosing $a$ appropriately (although the constants will change).

\section{Van der Corput lemmas}

As in a number of papers that give asymptotics for oscillatory integrals and related matters, we will make significant use of lemmas related to the classical Van der Corput lemma. When $K=\mathbf{R}$ what we will need will readily follow from the classical one-dimensional Van der Corput lemma:

Classical Van der Corput Lemma. Suppose $g(x)$ is a $k$ times differentiable function on an interval $I$ satisfying $\left|g^{(k)}(t)\right|>\eta>0$ for all $t$. Then for a constant $A_{k}$ depending only on $k$, for any $\epsilon>0$ we have

$$
|\{t \in I:|g(t)|<\epsilon\}|<A_{k} \epsilon^{\frac{1}{k}} \eta^{-\frac{1}{k}}
$$

If $\phi(x)$ is a $C^{1}$ function on $I$ and $k>1$, then for some constant $B_{\phi, k}$ one has

$$
\left|\int_{I} e^{i \lambda g(t)} \phi(t) d t\right|<B_{\phi, k}(1+|\lambda|)^{-\frac{1}{k}}
$$

If $g^{\prime}(t)$ is piecewise monotone on $I$, then $(2.1 b)$ also holds for $k=1$, where the constant will now also depend on the number of pieces on which $g^{\prime}(t)$ is monotone.

For $K$ other than $\mathbf{R}$, we will also make use of versions of the Van der Corput lemma that hold for analytic functions. The sublevel set version we will use can be stated as follows.

Theorem 2.1. Suppose $U \subset K^{n}$ is a bounded open set with $0 \in U$, and suppose $f(x)$ is a function whose Taylor series at the origin converges on a neighborhood of $c l(U)$. Suppose 
also that there is some $k>0$ such that $\left|\partial_{x_{n}}^{k} f(x)\right| \neq 0$ on $\operatorname{cl}(U)$. Then there is a constant $B$ independent of $\epsilon$ (but depending on $f(x)$ ) such that for all $\epsilon>0$ one has

$$
|\{x \in U:|f(x)|<\epsilon\}|<B \epsilon^{\frac{b_{K}}{k}}
$$

Proof. The case where $K=\mathbf{R}$ follows immediately by localizing and then applying the Van der Corput Lemma in the $x_{n}$ direction. Suppose now $K=\mathbf{C}$. It suffices to prove (2.2) in a neighborhood of any $x_{0} \in c l(U)$. If $f\left(x_{0}\right) \neq 0$, this is immediate. So we suppose $f\left(x_{0}\right)=0$. Let $l>0$ be minimal such that $\partial_{x_{n}}^{l} f\left(x_{0}\right)=0$; by assumption, $l \leq k$. Let $F(x)=f\left(x_{0}+x\right)$. By the Weierstrass preparation theorem, there is an open set $V$ containing 0 on which we may write

$$
F(x)=c(x)\left(x_{n}^{l}+\sum_{i=0}^{l-1} a_{i}\left(x_{1}, \ldots, x_{n-1}\right) x_{n}^{i}\right)
$$

Here $c(x)$ is analytic with $c(0) \neq 0$. Since $c(0) \neq 0$, shrinking $V$ if necessary it suffices to show that for a constant $C$ independent of $\epsilon$, for all $\epsilon>0$ we have

$$
\left|\left\{x \in V:\left|x_{n}^{l}+\sum_{i=0}^{l-1} a_{i}\left(x_{1}, \ldots, x_{n-1}\right) x_{n}^{i}\right|<\epsilon\right\}\right|<C \epsilon^{\frac{2}{k}}
$$

By the fundamental theorem of algebra we may factorize

$$
x_{n}^{l}+\sum_{i=0}^{l-1} a_{i}\left(x_{1}, \ldots, x_{n-1}\right) x_{n}^{i}=\prod_{i=1}^{l}\left(x_{n}-\alpha_{i}\left(x_{1}, \ldots, x_{n-1}\right)\right)
$$

In order for the product on the right of (2.5) to be less than $\epsilon$ in magnitude, at least one $\left|x_{n}-\alpha_{i}\left(x_{1}, \ldots, x_{n-1}\right)\right|$ must be less than $\epsilon^{\frac{1}{\tau}}$. Hence given $\left(x_{1}, \ldots, x_{n-1}\right)$, the set of $x_{n}$ for which the magnitude of the product is less than $\epsilon$ has measure at most $l \epsilon^{\frac{2}{l}} \leq k \epsilon^{\frac{2}{k}}$. Integrating this over all $\left(x_{1}, \ldots, x_{n-1}\right) \in V$ gives $(2.4)$ as needed. This completes the proof for $K=\mathbf{C}$.

If $K$ is a $p$-adic field, we do a similar argument using the $p$-adic Weierstrass preparation theorem (see Theorem 6.2.10 of [Go]). As in the complex case, we may focus our attention on an $x_{0} \in \operatorname{cl}(U)$ for which $f\left(x_{0}\right)=0$, and we define $F(x)=F\left(x_{0}+x\right)$. Again let $l$ be minimal such that $\partial_{x_{n}}^{l} f\left(x_{0}\right)=0$. By the $p$-adic Weierstrass preparation theorem, there are balls $B_{1} \subset K$ and $B_{2} \subset K^{n-1}$ centered at the origins of $K$ and $K^{n-1}$ respectively such that if $\left(x_{1}, \ldots, x_{n-1}\right) \in B_{2}$, then there are $b_{0}\left(x_{1}, \ldots, x_{n-1}\right), \ldots, b_{l-1}\left(x_{1}, \ldots, x_{n-1}\right) \in K$ and a constant $A>0$ such that for $x_{n} \in B_{1}$ one has

$$
|F(x)|=A\left|x_{n}^{l}+\sum_{i=0}^{l-1} b_{i}\left(x_{1}, \ldots, x_{n-1}\right) x_{n}^{i}\right|
$$


Hence given $\left(x_{1}, \ldots, x_{n-1}\right) \in B_{2}$, there is a finite extension $L$ of $K$ and $\beta_{i}\left(x_{1}, \ldots, x_{n-1}\right) \in L$ such that

$$
|F(x)|=A\left|\prod_{i=1}^{l}\left(x_{n}-\beta_{i}\left(x_{1}, \ldots, x_{n-1}\right)\right)\right|
$$

Thus if $|F(x)|<\epsilon$, there is some $i$ for which $\left|x_{n}-\beta_{i}\left(x_{1}, \ldots, x_{n-1}\right)\right|<A^{-\frac{1}{\tau}} \epsilon^{\frac{1}{\tau}}$ in $L$. By the ultrametric property of $p$-adic fields, if there is any $y \in K$ such that $\left|y-\beta_{i}\left(x_{1}, \ldots, x_{n-1}\right)\right|<$ $A^{-\frac{1}{l}} \epsilon^{\frac{1}{l}}$, then for all $z \in K$ with $\left|z-\beta_{i}\left(x_{1}, \ldots, x_{n-1}\right)\right|<A^{-\frac{1}{l}} \epsilon^{\frac{1}{l}}$ one has $|z-y|<A^{-\frac{1}{l}} \epsilon^{\frac{1}{l}}$ as well. Hence either the set of $x_{n} \in B_{1}$ for which $\left|x_{n}-\beta_{i}\left(x_{1}, \ldots, x_{n-1}\right)\right|<A^{-\frac{1}{l}} \epsilon^{\frac{1}{l}}$ is empty, or it is a subset of the set of $x_{n} \in B_{1}$ for which $\left|x_{n}-y_{i}\left(x_{1}, \ldots, x_{n-1}\right)\right|<A^{-\frac{1}{\tau}} \epsilon^{\frac{1}{\tau}}$, where $y_{i}\left(x_{1}, \ldots, x_{n-1}\right)$ is some element of $K$. This set has measure bounded by $A^{\frac{-b_{K}}{l}} \epsilon^{\frac{b_{K}}{l}}$. Hence for our fixed $\left(x_{1}, \ldots, x_{n-1}\right)$, the set of $x_{n} \in B_{1}$ with $|F(x)|<\epsilon$ has measure at most $A^{\frac{-b_{K}}{l}} l \epsilon^{\frac{b_{K}}{l}} \leq A^{\frac{-b_{K}}{l}} k \epsilon^{\frac{b_{K}}{k}}$. Like with $K=\mathbf{C}$, we now integrate this over all $\left(x_{1}, \ldots, x_{n-1}\right) \in$ $B_{2}$, obtaining (2.2) as needed. This completes the proof of Theorem 2.1.

For the oscillatory integrals (1.1), (1.3), (1.5) the analogue to Theorem 2.1 holds as well. For $K=\mathbf{R}$ this is once again an immediate consequence of the Van der Corput lemma. When $K$ is a $p$-adic field, it is a consequence of a recent result of Cluckers [Cl]; see also $[\mathrm{R}]$ for an earlier partial result. The proof in $[\mathrm{Cl}]$ appears to extend also to $K=\mathbf{C}$. For the purposes of our paper however we need the following result which relies on resolution of singularities:

Theorem 2.2. Suppose $f(x)=\sum_{\alpha} f_{\alpha} x^{\alpha}$ converges on a neighborhood of the origin in $K^{n}$ with $f(0)=0$. Suppose $\delta$ and $l$ are such that for any sufficiently small open $U$ containing the origin, for all $0<\epsilon<\frac{1}{2}$ one has

$$
|\{x \in U:|f(x)|<\epsilon\}|<A_{f, U} \epsilon^{\delta}|\ln (\epsilon)|^{l}
$$

Then there is a neighborhood $V$ of the origin such that if the support of the integrand of the oscillatory integral (1.1), (1.3), or (1.5) is contained in $V$, then for sufficiently large $|\lambda|,|w|$, or $|z|$ respectively the oscillatory integral satisfies the analogous bound $|I(\lambda)| \leq C(\ln |\lambda|)^{l}|\lambda|^{-\delta},|I(w)| \leq C(\ln |w|)^{l}|w|^{-\delta}$, or $|I(z)| \leq C(\ln |z|)^{l}|z|^{-\delta}$.

Proof. When $K=\mathbf{R}$, Theorem 2.2 is a relatively straightforward consequence of the existence of asymptotic expansions for sublevel set measures and oscillatory integrals; we refer to $[\mathrm{AGV}] \mathrm{Ch} 7$ for details. An elementary proof based on an earlier version of the resolution of singularities algorithm of [G4] was also given in [G2]. When $K$ is a $p$-adic field, Theorem 2.2 was shown by Igusa [I1]-[I3].

It remains to consider $K=\mathbf{C}$. We will prove Theorem 2.2 in the complex case using results from [G4] (and it can be proved in a very similar fashion using Hironaka's theorem [Hi1]-[Hi2]); the proof will also be quite similar to aforementioned argument of [I1]-[I3] for the $p$-adic field case. So suppose $K=\mathbf{C}$ now. By Theorem 1.1 of [G4], there are a finite collection of functions $\beta_{m}(z)$ such that if $\phi(x)$ is a cutoff function supported 
on a sufficiently small neighborhood of the origin, one can write $\phi(x)=\sum_{m=1}^{N} \tilde{\phi}_{m}(x)$ such that each $\phi_{m}(z)=\tilde{\phi} \circ \beta_{m}(z)$ can be changed on a set of measure zero to become a smooth function. Furthermore each $\beta_{m}(z)$ is one-to-one almost everywhere and on the support of $\phi_{m}$, and both $f \circ \beta_{m}(z)$ and the Jacobian $J_{m}(z)$ of $\beta_{m}(z)$ are of the form $a_{m}(z) p_{m}(z)$ with $a_{m}(z)$ nonvanishing and $p_{m}(z)$ a monomial. So for any measurable bounded function $h(x)$ we have

$$
\int h(x) \phi(x) d x=\sum_{m=1}^{N} \int h \circ \beta_{m}(z)\left|J_{m}(z)\right|^{2} \phi_{m}(z) d z
$$

Here $\phi_{m}(z)$ is smooth and on the support of the integrand of a given term of the right-hand side of (2.9) the function $f \circ \beta_{m}(z)$ is of the form $a_{m}(z) p_{m}(z)$ where $a_{m}(z)$ is nonvanishing and $p_{m}(z)$ is a nonconstant monomial. Applying (2.9) to $h(x)=e^{i R e(w f(x))}$ for $w \in \mathbf{C}$ we get

$$
\int e^{i \operatorname{Re}(w f(x))} \phi(x) d x=\sum_{m=1}^{N} \int e^{i \operatorname{Re}\left(w a_{m}(z) p_{m}(z)\right)}\left|J_{m}(z)\right|^{2} \phi_{m}(z) d z
$$

Since we may restrict ourselves to $x$ in an arbitrarily small neighborhood of the origin, in a given term of (2.10) we may restrict ourselves to $z$ in an arbitrarily small neighborhood of $\beta_{m}^{-1}(0)$. Write $p_{m}(z)=\prod_{i=1}^{n} z_{i}^{q_{i}}$. If $z \in \beta_{m}^{-1}(0)$, then since $f(0)=0$ one has $f \circ \beta_{m}(z)=$ $f(0)=0$ and therefore $p_{m}(z)=0$. As a result, $\beta_{m}^{-1}(0)$ is a subset of $\left\{z \in \mathbf{C}^{n}: z_{i}=0\right.$ for some $i$ with $\left.q_{i}>0\right\}$. Using a partition of unity, splitting $\phi_{m}$ into a finite sum of bump functions with smaller support as needed, without loss of generality we can assume for some $z^{\prime} \in \beta_{m}^{-1}(0)$ that $\phi_{m}$ is supported on a small neighborhood of $z^{\prime}$ such that for some $i^{\prime}$ with $z_{i}^{\prime}=0$ and $q_{i}>0$, we can do a change of variables such that what was once $a_{m}(z) z_{i}^{q_{i}}$ becomes $z_{i}^{q_{i}}$. (Recall that $a_{m}(z)$ is nonvanishing). Thus in place of a given term of $(2.10)$ we may focus on

$$
\int e^{i \operatorname{Re}\left(w \prod_{i=1}^{n} z_{i}^{q_{i}}\right)}\left|J_{m}(z)\right|^{2} \phi_{m}(z) d z
$$

Note that $\phi_{m}(z)$ may be a different bump function from that of (2.10) due to the localization and variable change. Changing variable names if necessary we can assume $q_{1}>0$ in (2.11). The idea now will be to use real integrations by parts in the $z_{1}$ variable in (2.11) and then integrate the result in the remaining variables. To this end, for fixed $\left(z_{2}, \ldots, z_{n}\right)$ we do a variable change of the form $z_{1} \rightarrow e^{i \theta} z_{1}$ so that the integral of the integrand of (2.11) in the $z_{1}$ variable becomes of the form

$$
\int e^{i \operatorname{Re}\left(|w|\left(\prod_{i=2}^{n}\left|z_{i}\right|^{q_{i}}\right) z_{1}^{q_{1}}\right)}\left|J_{m}(z)\right|^{2} \tilde{\phi}_{m}(z) d z_{1}
$$

We divide this dyadically as

$$
\sum_{j} \int e^{i R e\left(|w|\left(\prod_{i=2}^{n}\left|z_{i}\right|^{q_{i}}\right) z_{1}^{q_{1}}\right)}\left|J_{m}(z)\right|^{2} \phi_{m j}(z) d z_{1}
$$

Here $\phi_{m j}(z)$ is supported in $2^{-j-1}<\left|z_{1}\right|<2^{-j+1}$. Observe that for fixed $\left(z_{2}, \ldots, z_{n}\right)$ the phase in $(2.12 b)$ is of the form $A R e\left(z_{1}^{q_{1}}\right)$, which is a homogeneous polynomial of degree $q_{1}$ 
with gradient of magnitude $\sim A\left|z_{1}\right|^{q_{1}-1}$. Hence one can integrate by parts as many times as one wants; each time one gains a factor of $\frac{C}{A\left|z_{1}\right|^{q_{1}-1}}$ but one also loses a factor of $\frac{C^{\prime}}{\left|z_{1}\right|}$ each time a derivative lands on $\left|J_{m}(z)\right|^{2}$ or $\phi_{m j}(z)$. Hence the net effect of $N$ integrations by parts is a factor of $\frac{C_{N}}{A^{N}\left|z_{1}\right|^{q_{1}} N}$. As a result, the $j$ th term of $(2.12 b)$ is bounded by

$$
C_{N} \int_{\left(z_{1}, \ldots, z_{n}\right) \in \operatorname{supp}\left(\phi_{m j}\right)}\left(|w| \prod_{i=1}^{n}\left|z_{i}\right|^{q_{i}}\right)^{-N}\left|J_{m}(z)\right|^{2} d z_{1}
$$

By taking absolute values of the integrand and then integrating, the $j$ th term of $(2.12 b)$ is also bounded by

$$
C \int_{\left(z_{1}, \ldots, z_{n}\right) \in \operatorname{supp}\left(\phi_{m j}\right)}\left|J_{m}(z)\right|^{2} d z_{1}
$$

Combining (2.13) and (2.14), and then adding the result over all $j$, we see that $(2.12 a)$ is bounded by

$$
C_{N}^{\prime} \int_{\left(z_{1}, \ldots, z_{n}\right) \in \operatorname{supp}\left(\phi_{m}\right)} \min \left(1,\left(|w| \prod_{i=1}^{n}\left|z_{i}\right|^{q_{i}}\right)^{-N}\right)\left|J_{m}(z)\right|^{2} d z_{1}
$$

Integrating this bound in the $z_{2}, \ldots, z_{n}$ variables, we get that $(2.11)$ is bounded by

$$
C_{N}^{\prime} \int_{\text {supp }\left(\phi_{m}\right)} \min \left(1,\left(|w| \prod_{i=1}^{n}\left|z_{i}\right|^{q_{i}}\right)^{-N}\right)\left|J_{m}(z)\right|^{2} d z_{1} \ldots d z_{n}
$$

As long as $N$ is sufficiently large, by Lemma 3.2a) of [G3] for example, the integral (2.16) will be bounded by a constant times the integral over the portion of the domain where $|w| \prod_{i=1}^{n}\left|z_{i}\right|^{q_{i}}<1$, in other words the portion where $\prod_{i=1}^{n}\left|z_{i}\right|^{q_{i}}<\frac{1}{|w|}$. Since $f \circ \beta_{m}(z)$ is within a constant factor of $\prod_{i=1}^{n}\left|z_{i}\right|^{q_{i}}$ on the support of $\phi_{m}(z)$, if $N$ is taken large enough (2.16) is at most

$$
C_{N}^{\prime \prime} \int_{\left\{z \in \operatorname{supp}\left(\phi_{m}\right): f \circ \beta_{m}(z)<\frac{1}{|w|}\right\}}\left|J_{m}(z)\right|^{2} d z
$$

If we let $h(x)$ be the characteristic function of the set where $|f(x)|<\frac{1}{|w|}$ in $(2.9)$, we see by adding (2.17) over all $m$ that (2.11) is at most a constant times the measure of the portion of the support of $\phi(x)$ where $|f(x)|<\frac{1}{|w|}$. By (2.8) this is at most $A_{U}(1+|w|)^{-\delta} \ln (1+|w|)^{l}$ and we are done with the proof.

Note that although Theorems 2.1 and 2.2 taken together imply the analogue of Theorem 2.1 for oscillatory integrals, one does not get a uniform constant this way as in the classical Van der Corput lemma. So in particular we do not recover the whole $p$-adic Van der Corput lemma of [Cl].

\section{Proofs of Theorems 1.1, 1.2, 1.3, and 1.4.}


We will make use of the following lemma from [G4].

Lemma 3.1. (Lemma 3.5 of [G4]). Let $K_{0}=K-\{0\}$ as before. For $0 \leq i \leq n-1$, let $\left\{F_{i j}\right\}$ denote the faces of $N(f)$ of dimension $i$. Let $o_{i j}$ denote the maximum order of the zeroes of $f_{F_{i j}}(x)$ on $K_{0}^{n}$ (see Definition 1.5). Then for each $i$ and $j$ there are finitely many maps $\gamma_{i j k}(x)$, each a composition of blowups (and therefore a bijective monomial map when restricted to $\left.K_{0}^{n}\right)$, such that the following holds.

There is an open $E$ containing the origin and constants $a, b, b^{\prime}>0$ such that if $\phi(x) \in C_{c}(E)$, outside a set of measure zero one can write $\phi(x)=\sum_{i j k} \phi_{i j k}(x)$, where each $\phi_{i j k}$ is supported in $E$ and each $\rho_{i j k}=\phi_{i j k} \circ \gamma_{i j k}$ is a function on $K_{0}^{n}$ extending to a smooth function on all of $K^{n}$. This can be done in such a way that the following hold.

a) Suppose $i>0$. Let $B(0, a)$ denote $\left\{y:\left|y_{l}\right|<a\right.$ for all $\left.1 \leq p\right\}$ and let $Z_{i j k}=\{z: a<$ $\left|z_{m}\right|<2$ for all $\left.1 \leq m \leq n-p\right\}$. Then for some $p$ there exists an open $Z_{i j k}^{\prime} \subset Z_{i j k}$ such that

$$
B(0, a) \times Z_{i j k}^{\prime} \subset \operatorname{supp}\left(\rho_{i j k}\right) \subset B(0,2) \times Z_{i j k}
$$

Furthermore, if $m(x)$ denotes $x^{v}$ for any vertex $v$ of $N(f)$ on $F_{i j}$, then there exists a directional derivative $\sum_{m} \beta_{m} \partial_{z_{m}}$ with $\sum_{m}\left|\beta_{m}\right|=1$ and and an integer $0 \leq q \leq o_{i j}$ such that on $\operatorname{supp}\left(\rho_{i j k}\right)$ one has

$$
b\left|m \circ \gamma_{i j k}(y, z)\right| \leq\left|\left(\sum_{m} \beta_{m} \partial_{z_{m}}\right)^{q}\left(f \circ \gamma_{i j k}\right)(y, z)\right| \leq b^{\prime}\left|m \circ \gamma_{i j k}(y, z)\right|
$$

b) Suppose $i=0$, so that $F_{0 j}$ is a single vertex $v$. Then we have

$$
B(0, a) \subset \operatorname{supp}\left(\rho_{0 j k}\right) \subset B(0,2)
$$

If $m(x)=x^{v}$ then on $\operatorname{supp}\left(\rho_{0 j k}\right)$ one has

$$
b\left|m \circ \gamma_{0 j k}(y)\right| \leq\left|f \circ \gamma_{0 j k}(y)\right| \leq b^{\prime}\left|m \circ \gamma_{0 j k}(y)\right|
$$

Let $\phi(x)$ be a cutoff function satisfying the hypotheses of Lemma 3.1. Then by the change of variables formulas (which holds for any $K$ ), if $j(x)$ is any bounded measurable function defined on the support of $\phi(x)$ we have

$$
\begin{aligned}
& \int j(x) \phi(x) d x=\sum_{i j k} \int j(x) \phi_{i j k}(x) d x \\
= & \sum_{i j k} \int j \circ \gamma_{i j k}(x)\left|J_{i j k}(x)\right|^{b_{K}} \rho_{i j k}(x) d x
\end{aligned}
$$


Here $J_{i j k}(x)$ denotes the Jacobian determinant of $\gamma_{i j k}(x)$, which is a monomial since each component of $\gamma_{i j k}(x)$ is.

By Lemma 3.3 of [G4], for a given $(i, j, k)$ with $i>0$ there is a single $\left(a_{1}, \ldots, a_{p}\right)$ such that if $m(x)=x^{v}$ for any vertex $v$ of $N(f)$ on $F_{i j}$, then $m \circ \gamma_{i j k}(x)$ is of the form $y_{1}^{a_{1}} \ldots y_{p}^{a_{p}} z_{1}^{b_{1}} \ldots z_{n-p}^{b_{n-p}}$ for some $\left(b_{1}, \ldots, b_{n-p}\right)$ which depends on $v$. When $i=0$, we define the $a_{l}$ 's by $y_{1}^{a_{1}} \ldots y_{n}^{a_{n}}=m \circ \gamma_{0 j k}(x)$, where $m(x)=x^{v}$ for $v$ the vertex of $N(f)$ corresponding to $F_{0 j}$. The next lemma relates the $a_{l}$ 's to $J_{i j k}(x)$ and the Newton distance $d(f)$.

Lemma 3.2. Let $h$ be the dimension of the central face of $N(f)$ as in the statement of Theorems 1.1-1.4. Write the Jacobian determinant $J_{i j k}(x)$ of $\gamma_{i j k}(x)$ as $y_{1}^{e_{1}} \ldots y_{p}^{e_{p}} z_{1}^{f_{1}} \ldots z_{n-p}^{f_{n-p}}$ (with only $y$ variables if $i=0$ ). Then for all $1 \leq l \leq p$ one has

$$
\frac{a_{l}}{e_{l}+1} \leq d(f)
$$

Equality holds for at most $n-h$ values of $l$, and there is at least one $(i, j, k)$ such that equality does hold for $n-h$ values of $l$. Equality can only hold if $F_{i j}$ is a subset of the central face of $N(f)$.

Proof. In [G4], the set of all $\left(a_{1}, \ldots, a_{p}\right)$ and $\left(e_{1}, \ldots, e_{p}\right)$ was determined by $N(f)$ and not the particular field $K$. Hence without loss of generality we may take $K=\mathbf{R}$. If one does the coordinate change $y_{l}=Y_{l}^{\frac{1}{e_{l}+1}}$ and $z_{m}=Z_{m}^{\frac{1}{f_{m}+1}}$ for all $l, m$ and let $\Gamma_{i j k}(Y, Z)$ be the composition of $\gamma_{i j k}(x)$ with this coordinate change, then $\Gamma_{i j k}(Y, Z)$ has constant Jacobian determinant and furthermore if $m$ is as in Lemma 3.1 we have

$$
m \circ \Gamma_{i j k}(Y, Z)=Y_{1}^{\frac{a_{1}}{e_{1}+1}} \ldots Y_{p}^{\frac{a_{p}}{e_{p}+1}} Z_{1}^{\frac{b_{1}}{f_{1}+1}} \ldots Z_{n-p}^{\frac{b_{n-p}}{f_{n-p}}}
$$

The lemma is now an immediate consequence of Lemma 2.6 of [G3].

In order to prove Theorem 1.1, we apply (3.3) with $j(x)$ the characteristic function of $\{x \in U:|f(x)|<\epsilon\}$ for a sufficiently small neighborhood $U$ of the origin. We will bound a given term $I_{i j k}$ of the sum (3.3) for this $j(x)$ and add the bounds. This will give Theorem 1.1. We first consider a term $I_{i j k}$ such that $i=0$ or such that $i>0$ but $q=0$ in $(3.1 b)$. In these situations, since the $\left|z_{m}\right|$ are bounded above and below, by $(3.1 b)-(3.2 b)$ there are constants $C, C^{\prime}>0$ such that on the domain of integration of $I_{i j k}$ one has

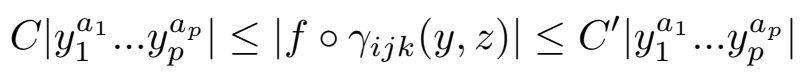

Similarly, the fact that the $\left|z_{m}\right|$ are bounded above and below imply that on the domain of integration of $I_{i j k}$ one has

$$
C^{\prime \prime}\left|y_{1}^{e_{1}} \ldots y_{p}^{e_{p}}\right| \leq\left|J_{i j k}(y, z)\right| \leq C^{\prime \prime \prime}\left|y_{1}^{e_{1}} \ldots y_{p}^{e_{p}}\right|
$$


In view of the inclusions $(3.1 a)-(3.2 a), I_{i j k}$ therefore satisfies

$$
\begin{aligned}
& C_{1} \int_{\left\{y \in B(0, a):\left|y_{1}^{a_{1}} . . y_{p}^{a_{p}}\right|<\frac{\epsilon}{C^{\prime}}\right\}}\left|y_{1}^{e_{1}} \ldots y_{p}^{e_{p}}\right|^{b_{K}} d y \leq I_{i j k}
\end{aligned}
$$

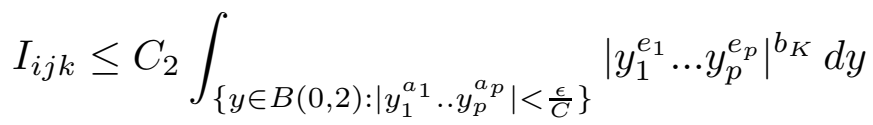

Suppose $K=\mathbf{R}$ now (so $b_{K}=1$ ), and change variables in (3.7), with the new $y_{j}$ being the former $y_{j}^{e_{j}+1}$. Then for some $a^{\prime}, b^{\prime}, c^{\prime}$, and $d^{\prime},(3.7)$ becomes

$$
C_{3} \int_{\left\{y \in B\left(0, a^{\prime}\right):\left|y_{1}^{\frac{a_{1}}{e^{+1}}} \ldots y_{p}^{\frac{a_{p}}{e_{p}+1}}\right|<c^{\prime} \epsilon\right\}} 1 d y \leq I_{i j k} \leq C_{4} \int_{\left\{y \in B\left(0, b^{\prime}\right):\left|y_{1}^{\frac{a_{1}}{e_{1}+1}} \ldots y_{p}^{\frac{a_{p}+1}{e_{p}}}\right|<d^{\prime} \epsilon\right\}} 1 d y
$$

Measures of sets of the form $\left\{y \in B(0,1):\left|y_{1}^{\frac{a_{1}}{e_{1}+1}} \ldots y_{p}^{\frac{a_{p}}{e_{p}+1}}\right|<\delta\right\}$ were analyzed in [G3]. By Lemma 3.1a) of [G3], if $M_{i j k}$ denotes the measure of this set, $\rho$ denotes the maximum of the $\frac{a_{l}}{e_{l}+1}$, and $\sigma$ denotes the number of times this value of $\frac{a_{l}}{e_{l}+1}$ occurs, we have

$$
C_{5} \delta^{\frac{1}{\rho}}|\ln (\delta)|^{\sigma-1} \leq M_{i j k} \leq C_{6} \delta^{\frac{1}{\rho}}|\ln (\delta)|^{\sigma-1}
$$

Thus using (3.9) and rescaling the balls of the left and right-hand sides of (3.8), we get

$$
C_{7} \epsilon^{\frac{1}{\rho}}|\ln (\epsilon)|^{\sigma-1} \leq I_{i j k} \leq C_{8} \epsilon^{\frac{1}{\rho}}|\ln (\epsilon)|^{\sigma-1}
$$

In the case where $K$ is a field other than $\mathbf{R}$, one can prove the analogous statement to (3.9) as in the proof of Lemma 3.1a) of [G3], inducting on the dimension and using Fubini's theorem. The analogue one obtains is

$$
C_{5}^{\prime} \delta^{\frac{b_{K}}{\rho}}|\ln (\delta)|^{\sigma-1} \leq M_{i j k} \leq C_{6}^{\prime} \delta^{\frac{b_{K}}{\rho}}|\ln (\delta)|^{\sigma-1}
$$

Thus we get the following generalization of (3.10).

$$
C_{7}^{\prime} \epsilon^{\frac{b_{K}}{\rho}}|\ln (\epsilon)|^{\sigma-1} \leq I_{i j k} \leq C_{8}^{\prime} \epsilon^{\frac{b_{K}}{\rho}}|\ln (\epsilon)|^{\sigma-1}
$$

Since by Lemma 3.2, $\frac{1}{\rho} \geq \frac{1}{d(f)}$ with $\sigma \leq n-h$ when $\rho=d(f)$, equation $\left(3.10^{\prime}\right)$ gives the required upper bounds for Theorem 1.1 for any $I_{i j k}$ with $i=0$, or with $i>0$ and $q=0$.

We now consider the terms $I_{i j k}$ of the sum (3.3) for $i>0$ such that $q>0$ in $(3.1 b)$. Doing a linear change of variables in the $z$ variables if necessary, without loss of generality we may assume that the directional derivative $\sum_{m} \beta_{m} \partial_{z_{m}}$ is just $\partial_{z_{n}}$. To find the needed upper bounds, we integrate the integrand of $I_{i j k}$ in the $z_{n}$ direction first, using Theorem 2.1, and then integrate the result in the remaining directions. Using (3.1b) in conjunction with Theorem 2.1, we see that this one-dimensional integral in the $z_{n}$ direction is bounded by $C \epsilon^{\frac{b_{K}}{q}}\left|y_{1}\right|^{-\frac{a_{1} b_{K}}{q}} \ldots\left|y_{p}\right|^{-\frac{a_{p} b_{K}}{q}}\left|z_{1}\right|^{-\frac{b_{1} b_{K}}{q}} \ldots\left|z_{n-p-1}\right|^{-\frac{b_{n-p-1} b_{K}}{q}}$. Since 
the $\left|z_{m}\right|$ are bounded away from 0 , this is bounded by $C^{\prime} \epsilon^{\frac{b_{K}}{q}}\left|y_{1}\right|^{-\frac{a_{1} b_{K}}{q}} \ldots\left|y_{p}\right|^{-\frac{a_{p} b_{K}}{q}}$. This one dimensional integral is also bounded by a uniform constant since the domain of integration is bounded. Integrating the minimum of these two bounds in the $\left(z_{1}, \ldots, z_{n-1}\right)$ variables, we obtain (using that $\left|J_{i j k}(y, z)\right| \sim\left|y_{1}^{e_{1}} \ldots y_{p}^{e_{p}}\right|$ since the $\left|z_{m}\right|$ are bounded above and below)

$$
I_{i j k} \leq C_{1} \int_{B(0,2)}\left|y_{1}^{e_{1}} \ldots y_{p}^{e_{p}}\right|^{b_{K}} \min \left(1, \epsilon^{\frac{b_{K}}{q}}\left|y_{1}\right|^{-\frac{a_{1} b_{K}}{q}} \ldots\left|y_{p}\right|^{-\frac{a_{p} b_{K}}{q}}\right) d y
$$

Again we first focus on the $K=\mathbf{R}$ case. Changing variables from $y_{j}$ to $y_{j}^{e_{j}+1}$, becomes

$$
I_{i j k} \leq C_{2} \int_{B(0,2)} \min \left(1, \epsilon^{\frac{1}{q}}\left|y_{1}\right|^{-\frac{a_{1}}{q\left(e_{1}+1\right)}} \ldots\left|y_{p}\right|^{-\frac{a_{p}}{q\left(e_{p}+1\right)}}\right) d y
$$

Integrals of the form (3.12) were also analyzed in [G3]. Again let $\rho=\max _{j} \frac{a_{j}}{e_{j}+1}$ and $\sigma$ the number of times this maximum is achieved. By Lemma 3.1 a) and d) of [G3], if $\rho>q$, one has

$$
I_{i j k} \leq C_{3} \epsilon^{\frac{1}{\rho}}|\ln (\epsilon)|^{\sigma-1}
$$

By Lemma 3.2 c) of [G3], if $\rho=q$ we have

$$
I_{i j k} \leq C_{4} \epsilon^{\frac{1}{\rho}}|\ln (\epsilon)|^{\sigma}
$$

While by Lemma 3.2b) of [G3], if $\rho<q$ then we have

$$
I_{i j k} \leq C_{5} \epsilon^{\frac{1}{q}}
$$

Note that the exponent of $\epsilon$ of $(3.13 a)-(3.13 c)$ can be succinctly written as $\min \left(\frac{1}{q}, \frac{1}{\rho}\right)$. In the case where $K$ is not $\mathbf{R}$, one can prove inequalities analogous to $(3.13 a)-(3.13 c)$ similarly to above; one may bound the right-hand side of (3.11) to obtain analogues to $(3.13 a)-(3.13 c)$ by inducting on the dimension and using Fubini's theorem. The result is the same as $(3.13 a)-(3.13 c)$, except the exponents of $\epsilon$ are multiplied by $b_{K}$. In other words, the exponent becomes $\min \left(\frac{b_{K}}{q}, \frac{b_{K}}{\rho}\right)$. Note that this exponent is decreasing with increasing $q$. Thus if $s(f)$ denotes the maximum value of $q$ over all faces of $N(f)$, this exponent is at least $\min \left(\frac{b_{K}}{s(f)}, \frac{b_{K}}{\rho}\right)$. Furthermore by Lemma $3.2, \rho \leq d(f)$, so that the exponent is at least $\min \left(\frac{b_{K}}{s(f)}, \frac{b_{K}}{d(f)}\right)$. Also, by Lemma 3.2 if $\rho=d(f)$ then $\sigma \leq n-h$, where $h$ is the dimension of the central face of $N(f)$. Equality may hold only when $F_{i j}$ is a subset of the central face of $N(f)$. Hence when $s(f)<d(f)$ we have

$$
I_{i j k} \leq C_{6} \epsilon^{\frac{b_{K}}{d(f)}}|\ln (\epsilon)|^{n-h-1}
$$

When $s(f)=d(f)$ we get

$$
I_{i j k} \leq C_{7} \epsilon^{\frac{b_{K}}{d(f)}}|\ln (\epsilon)|^{n-h}
$$

Here equality can only hold if $F_{i j}$ is a subset of the central face. When $s(f)>d(f)$ we get

$$
I_{i j k} \leq C_{8} \epsilon^{\frac{b_{K}}{s(f)}}
$$


These are exactly the exponents of Theorem 1.1. So adding over all $i, j$, and $k$ proves all upper bounds of Theorem 1.1. Now Theorem 1.2 follows immediately via Theorem 2.2.

The lower bounds of Theorem 1.1 hold for the following reason. By Lemma 3.2, there is at least one face $F_{i j}$ for which $\rho=d(f)$ and $\sigma=n-h$. The constructions of [G4] are such that for $F_{i j}$ one can ensure that there is at least one $k$ for which $(3.2 b)$ holds or for which $(3.1 b)$ holds with $q=0$. As a result, the lower bounds of $\left(3.10^{\prime}\right)$ imply the required lower bounds of Theorem 1.1, completing the proof of that theorem.

\section{Proof of Theorems 1.3 and $\mathbf{1 . 4 .}$}

Theorems 1.3 and 1.4 are translations of Theorems 1.1 and 1.2 respectively in the case when $K=\mathbf{Q}_{p}$, taking $\epsilon=p^{-l}$ in Theorem 1.1 and $z=p^{-l}$ in Theorem 1.2. If each $a_{i}$ is sufficiently large, by scaling the results for $f(x)$, Theorems 1.1 and 1.2 will hold for $f_{a}(x)$ on all of $\left\{x:\left|x_{i}\right| \leq 1\right.$ for all $\left.i\right\}$. If $\xi(z)$ denotes the standard character where $\xi\left(\sum_{k \geq k_{0}} b_{k} p^{k}\right)=e^{2 \pi i\left(\sum_{k=k_{0}}^{-1} b_{k} p^{k}\right)}$ if $k_{0}<0$ and $\xi(z)=1$ if $k_{0} \geq 0$, then since $f_{a}(x)$ has integer coefficients, $\xi\left(p^{-l} f_{a}(x)\right)$ will be constant on balls of radius $p^{-l}$. Thus

the integral $I\left(p^{-l}\right)=\int_{\left\{x:\left|x_{i}\right|<1 \forall i\right\}} \xi\left(p^{-l} f_{a}(x)\right) d x$ will be the average of the $p^{l n}$ different values $\xi\left(p^{-l} f_{a}(x)\right)$ achieves as each $x_{i}$ goes through the $p^{l}$ different balls of radius $p^{-l}$ i.e. through the different residue classes $\bmod p^{l}$. Thus Theorem 1.2 gives Theorem 1.4.

Similarly, in Theorem 1.1 the $x$ for which $\left|f_{a}(x)\right|<p^{-l}$ are exactly the $x$ such that $p^{l}$ divides $f_{a}(x)$ (viewing an element of $\mathbf{Q}_{p}$ as an infinite series of powers of $p$ ). Since $f(z)$ has integer coefficients, whenever $\left|f_{a}(x)\right|<p^{-l}$ one will also have that $\left|f_{a}\left(x^{\prime}\right)\right|<p^{-l}$ for any $x^{\prime}$ such that each $x_{i}^{\prime}=x_{i} \bmod p^{l}$. Thus whether or not $x$ satisfies $\left|f_{a}(x)\right|<p^{-l}$ depends on what each $x_{i} \bmod p^{l}$ is. Hence the bounds on the measure of the $x$ for which $\left|f_{a}(x)\right|<p^{-l}$, given by Theorem 1.1, translate into Theorem 1.3 and we are done.

Acknowledgement. The author would like to thank the referee for several helpful suggestions.

\section{References.}

[AGV] V. Arnold, S Gusein-Zade, A Varchenko, Singularities of differentiable maps Volume II, Birkhauser, Basel, 1988.

[Cl] R. Cluckers, Analytic Van der Corput Lemma for p-adic oscillatory integrals, singular Fourier transforms, and restriction theorems, Expositiones Mathematicae 29 (2011) No. 4, 371-386.

[D] J. Denef, Report on Igusa's local zeta function, Sminaire Bourbaki, Vol. 1990/91. Astrisque No. 201-203 (1991), Exp. No. 741, 359-386 (1992).

[DHo] J. Denef, K. Hoornaert, Newton polyhedra and Igusa's local zeta function, J. Number Theory 89 (2001), no. 1, 31-64.

[DLo] J. Denef, F. Loeser, Motivic Igusa zeta functions, J. Algebraic Geom. 7 (1998), no. $3,505-537$. 
[Go] F. Gouvea, p-adic numbers. An introduction, Second edition. Universitext. SpringerVerlag, Berlin, 1997. vi+298 pp.

[G1] M. Greenblatt, A Coordinate-dependent local resolution of singularities and applications, J. Funct. Anal. 255 (2008), no. 8, 1957-1994.

[G2] M. Greenblatt, Resolution of singularities, asymptotic expansions of oscillatory integrals, and related Phenomena, J. Analyse Math. 111 (2011) no. 1, 221-245.

[G3] M. Greenblatt, Oscillatory integral decay, sublevel set growth, and the Newton polyhedron, Math. Annalen 346 (2010), no. 4, 857-895.

[G4] M. Greenblatt, A constructive elementary method for local resolution of singularities, submitted.

[H1] H. Hironaka, Resolution of singularities of an algebraic variety over a field of characteristic zero I, Ann. of Math. (2) 79 (1964), 109-203.

[H2] H. Hironaka, Resolution of singularities of an algebraic variety over a field of characteristic zero II, Ann. of Math. (2) 79 (1964), 205-326.

[I1] J. Igusa, Complex powers and asymptotic expansions I, J. Reine Agnew. Math, 268/269 (1974) 110-130.

[I2] J. Igusa, Complex powers and asymptotic expansions II, J. Reine Agnew. Math, $\mathbf{2 7 8 / 2 7 9 ~ ( 1 9 7 5 ) ~ 3 0 7 - 3 2 1 . ~}$

[I3] J. Igusa, Lectures on forms of higher degree, Tata Inst. Fund. Research, Bombay, 1978.

[LM] B. Lichtin, D. Meuser, Poles of local zeta functions and Newton polygons, Compositio Math. 55 (1985), 313-332.

[R] K. Rogers, A van der Corput lemma for the p-adic numbers, Proc. Amer. Math. Soc. 133 (2005), no. 12, 3525-3534.

[V] A. N. Varchenko, Newton polyhedra and estimates of oscillatory integrals, Functional Anal. Appl. 18 (1976), no. 3, 175-196.

[Ve] W. Veys, Determination of the poles of the topological zeta function for curves, Manuscripta Math. 87 (1995), no. 4, 435-448.

[Wr] J. Wright, Exponential sums and polynomial congruences in two variables: the quasihomogeneous case, preprint.

[Zu1] W. Zuniga-Galindo, Igusa's local zeta functions of semiquasihomogeneous polynomials, Trans. Amer. Math. Soc. 353 (2001), no. 8, 3193-3207.

[Zu2] W. Zuniga-Galindo, Local zeta functions and Newton polyhedra, Nagoya Math. J. 172 (2003), 31-58.

Department of Mathematics, Statistics, and Computer Science

University of Illinois at Chicago

322 Science and Engineering Offices

851 S. Morgan Street

Chicago, IL 60607-7045

greenbla@uic.edu 